\section{Nuclear receptor in the crosshairs of diabetes}

\section{By Kai-Jye Lou, Staff Writer}

Researchers at the Baylor College of Medicine and Scripps Florida have identified a new signaling pathway that could be targeted to treat type 2 diabetes. ${ }^{1}$ The group has shown that activating the nuclear receptor LRH-1 pathway in mice can decrease fatty liver and increase insulin sensitivity without the typical weight gain caused by marketed PPAR agonists. The group's collaborators are running a pilot trial in prediabetic individuals to evaluate one LRH-1 agonist-dilauroyl phosphatidylcholine.

LRH-1 (nuclear receptor subfamily 5 group A member 2; NR5A2) is an orphan nuclear receptor best known for its role as a regulator of bile acid synthesis. ${ }^{2}$ Because high levels of bile acids in the liver are known to reduce fatty liver ${ }^{3}$ and improve glucose homeostasis, ${ }^{4}$ the BCM and Scripps team began a search for compounds that activate LRH-1.

Previous reports had also shown that LRH-1 could potentially interact with phospholipids, ${ }^{5,6}$ which led the researchers to focus their screening efforts on phospholipids that activated LRH-1.

Those screens turned up a pair of phospholipids-dilauroyl phosphatidylcholine (DLPC) and diundecanoyl phosphatidylcholine (DUPC) - as potent activators of LRH-1 signaling. Although the two compounds showed comparable activity in mouse and human cell lines, David Moore, a professor in the Department of Molecular and Cellular Biology at BCM, said DLPC was selected for further evaluation because its structure more closely resembles that of natural phospholipids.

Phosphatidylcholines are a major component of cellular membranes and are found in many common plant- and animal-derived food products, such as soybeans and eggs.

In two mouse models of insulin resistance, DLPC increased bile acid levels, reduced fatty liver and improved glucose homeostasis compared with vehicle control. DLPC also decreased expression of sterol regulatory element binding transcription factor 1 (Srebf1; Srebp1), which mediates lipogenesis and is associated with fatty liver (see Figure 1, "Model for LRH-1-mediated regulation of glucose metabolism").

In addition, DLPC did not activate other nuclear receptors, includ- ing peroxisome proliferation activated receptors (Ppars), and did not affect body weight in mice.

Results were published in Nature.

"DLPC's mechanism of action focuses primarily on reducing fatty liver," said Moore, who is corresponding author on the paper. "Thus, we believe that individuals that have developed fatty liver-associated insulin resistance would be the group most likely to benefit from treatment with a compound like DLPC."

"The data suggest that DLPC functions as an insulin sensitizer, so it addresses the key defect in type 2 diabetes," said Mitchell Lazar, chief of the Division of Endocrinology, Diabetes and Metabolism and director of the Institute for Diabetes, Obesity and Metabolism at the Perelman School of Medicine at the University of Pennsylvania. "This is similar to compounds that target PPAR $\gamma$, such as thiazolidinediones, but it does not seem to have the weight gain problem associated with PPAR $\gamma$ compounds, so that would be a plus."

Because LRH-1 is highly enriched in both the gut and liver, Johan Auwerx, a professor of life sciences at the Swiss Federal Institute of Technology Lausanne also thinks it should be possible to specifically target these tissues with LRH-1 agonists like DLPC.

In addition, Auwerx and colleagues at Louis Pasteur University have previously reported that lowering LRH-1 signaling in mice results in greater susceptibility to intestinal inflammation. ${ }^{7}$

Thus, he said, "it may be of interest to test DLPC in other indications that are modulated by LRH-1-one that immediately comes to mind is inflammatory bowel disease." Auwerx added that it would be interesting to explore whether increasing dietary intake of DLPC could prevent the onset of conditions like metabolic syndrome.

Moore said collaborators at BCM already are evaluating DLPC as a dietary supplement in an investigator-led, double-blind, placebo-controlled pilot study in prediabetic individuals. The pilot trial is studying the effects of DLPC on glucose tolerance and is supported by a fund managed by St. Luke's Episcopal Hospital.

"We are also interested in developing a synthetic LRH-1 agonist as a drug, but we would then need to determine whether it would have an effect similar to DLPC," added Moore.

\section{New signaling insights}

The data reported in Nature open up a new signaling pathway to mine for therapeutic targets and molecules against type 2 diabetes and other metabolic disorders like nonalcoholic fatty liver. The findings also could provide new insights on ways to modulate phospholipid activity.

"This work is an emerging story in diabetes mechanisms that links signaling through the LRH-1 nuclear receptor to lipid control and glucose homeostasis," said Jeffrey Johnson, principal scientist in biology at Metabolex Inc. 
Figure 1. Model for LRH-1-mediated regulation of glucose metabolism. As reported by Lee et al., the phospholipid dilauroyl phosphatidylcholine (DLPC) increases bile acid synthesis and insulin sensitivity and leads to improved glucose homeostasis.

The researchers hypothesized that DLPC's beneficial effects stem from its ability to activate nuclear receptor subfamily 5 group A member 2 (NR5A2; LRH-1), which according to the model would suppress sterol regulatory element binding transcription factor 1 (SREBF1; SREBP1) activity, decrease fatty liver and promote insulin sensitivity. This DLPC-induced increase in insulin sensitivity results in lower serum insulin levels and will thus further reduce SREBP1 activity (purple arrows).

In the current model, SREBP1 is part of a positive regulatory loop whereby the transcription factor promotes lipogenesis and the development of fatty liver, which leads to decreased insulin sensitivity. The reduction in insulin sensitivity results in higher serum insulin levels, which further increases SREBP1 activity.

Figure adapted from Lee et al.

He said it was particularly interesting that the degree of saturation of the phospholipid fatty acid tails appears to determine the molecule's binding activity. In the study, phospholipids with 11- or 12-carbon fatty acid tails potently activated LRH-1, whereas those with other tail lengths could not.

"In classical phospholipid signaling, such as that seen with the phosphatidylinositols, it is generally the phosphate head group of the molecule that dictates its binding activity as opposed to the degree of saturation of its fatty acid chains," noted Brian Lavan, senior director in biology at Metabolex.

The biotech's MBX-2982, a G protein-coupled receptor 119 (GPR119) agonist, has completed a Phase II trial for type 2 diabetes.

Despite the end result of increasing insulin sensitivity, Lavan said the LRH-1 activation mechanism appears to be very different from that of PPAR agonists. "Activation of LRH-1 increases bile acid synthesis in the liver, which is quite differentiated from activating PPARs," he told SciBX.

"PPAR $\gamma$ ligands and drugs decrease insulin resistance primarily by redistributing lipids from muscle and liver to adipose tissue," said Barry Forman, professor and director of the Division of Gene Regulation and Drug Discovery at City of Hope. "As it stands, one might expect an LRH-1 agonist to have a more focused effect on the liver."

To the extent that this is the case, LRH-1 agonists and PPAR $\gamma$ drugs "work via different mechanisms and could be particularly effective and less toxic in combination therapy," he told SciBX.

For therapeutic development, researchers contacted by SciBX wanted to see additional work to elucidate DLPC's activity and its mechanism of action.

"It would be interesting to know if DLPC is really the endogenous ligand of LRH-1 and, if so, how it gets into cells and what its concentration is relative to what it needs to be to activate the LRH-1 receptor," said Lazar. "This would be very valuable both in terms of using DLPC or similar compounds as actual drugs, as well as in designing better drugs to target this pathway."

"To consolidate the fact that LRH-1 is mediating the observed effects, reproduction of the observations with a synthetic and selective LRH-1 agonist would be important," added Auwerx. "Also it may be worth it to look at whether genetic mutations in the LRH-1 locus are associated with metabolic diseases. Likewise, is there epidemiological evidence that high DLPC content in the diet protects against metabolic dysfunction?"

Ronald Margolis, senior advisor for molecular endocrinology at the National Institute of Diabetes and Digestive and Kidney Diseases, said it will be important to determine whether DLPC is having a direct effect on LRH-1 or acting via an indirect pathway on another target. He also wanted to know whether it is possible to deliver DLPC into the cell and into the cell nucleus in sufficient quantities to activate LRH-1.

He added that the safety of using DLPC in the therapeutic setting needs further testing. "As one begins to study DLPC and compounds like it, we will want to determine if DLPC, when given at high enough levels, will be toxic to the cell and how much activation of the LRH-1 receptor is acceptable before we start seeing side effects," he said. "The latter is one question that was not sufficiently addressed during the development of the first-generation PPAR agonists."

Moore noted that for the pilot study, DLPC is being dosed at levels that would help determine the phospholipid's tolerability and side-effect profile in humans.

"A problem arises if DLPC has limitations such as toxicity or pharmacokinetic issues. In that case, the usual approach would be to design analogs that target the same pathway to identify better second-generation compounds," Forman told SciBX. "My concern is that the pathway may not be fully elucidated, making the screening process potentially challenging." He added that it will be important to determine whether DLPC is acting directly or indirectly via LRH-1 and whether LRH-1 binding is relevant to the observed therapeutic effects.

Moore said his group is carrying out studies to elucidate DLPC's mechanism of action.

BCM has filed a patent application covering the composition of DLPC and related compounds and their use in metabolic disorders and inflammatory bowel disease (IBD). The IP is available for licensing.

Lou, K.-J. SciBX 4(23); doi:10.1038/scibx.2011.649

Published online June 9, 2011 


\section{ANALYSIS}

\section{TARGETS \& MECHANISMS}

\section{REFERENCES}

1. Lee, J.M. et al. Nature; published online May 25, 2011; doi:10.1038/nature10111

Contact: David D. Moore, Baylor College of Medicine, Houston, Texas

e-mail: moore@bcm.tmc.edu

2. Lee, Y.-K. \& Moore, D.D. Front. Biosci. 13, 5950-5958 (2008)

3. Watanabe, M. et al. J. Clin. Invest. 113, 1408-1418 (2004)

4. Ma, K. et al. J. Clin. Invest. 116, 1102-1109 (2006)

5. Krylova, I.N. et al. Cell 120, 343-355 (2005)

6. Ortlund, E.A. et al. Nat. Struct. Mol. Biol. 12, 357-363 (2005)

7. Coste, A. et al. Proc. Natl. Acad. Sci. USA 104, 13098-13103 (2007)
COMPANIES AND INSTITUTIONS MENTIONED

Baylor College of Medicine, Houston, Texas

City of Hope, Duarte, Calif.

Louis Pasteur University, Illkirch, France

Metabolex Inc., Hayward, Calif.

National Institute of Diabetes and Digestive and Kidney Diseases,

Bethesda, Md.

Perelman School of Medicine at the University of Pennsylvania,

Philadelphia, $\mathrm{Pa}$.

St. Luke's Episcopal Hospital, Houston, Texas

Scripps Florida, Jupiter, Fla.

Swiss Federal Institute of Technology Lausanne,

Lausanne, Switzerland 